\title{
Structure of Burnout among Omani Male and Female Teachers: Invariance of Structure across Gender
}

\author{
Maher Abu-Hilal,* Said Al dafri, Hashem Kilani, Ali Kazem, Ibrahim Qaryouti, \\ \& Hussein AlKharusi \\ Sultan Qaboos University, Sultanate of Oman
}

Received: $11 / 3 / 2018$

Accepted: $17 / 5 / 2018$

\begin{abstract}
Previous studies have supported the conceptualization that burnout consists of three aspects: emotional exhaustion, depersonalization and reduced personal accomplishment. When the proposed structure of burnout was tested in several studies, these three aspects were confirmed. However, several researchers found that depersonalization consists of two factors rather than one. The first factor is related to the job, while the second factor is related to students. The original factor structure (3-factor model) and the revised structure were tested by few Arab researchers. Recently, the Maslach Burnout Inventory (MBI) has been introduced to the Omani educational setting. This study aimed to test the two structures of MBI with a representative sample of Omani teachers $(\mathrm{N}=2446)$. Confirmatory factor analysis (CFA) revealed that the 4-factor model was superior and more parsimonious (CFI $=.919$, RMSEA $=.051$ ) than the 3-factor model $(\mathrm{CFI}=.887$, RMSEA $=.059)$. Three of the four factors had a high reliability coefficient (emotional exhaustion, $\alpha=.87$, personal accomplishment, $\mathrm{a}=.76$, depersonalizationjob, $\alpha=.79)$ while depersonalization of students had low reliability $(\alpha=.47)$. The latter seems to be culture specific.
\end{abstract}

Keywords: Burnout, factorial validity, reliability, Omani teacher.

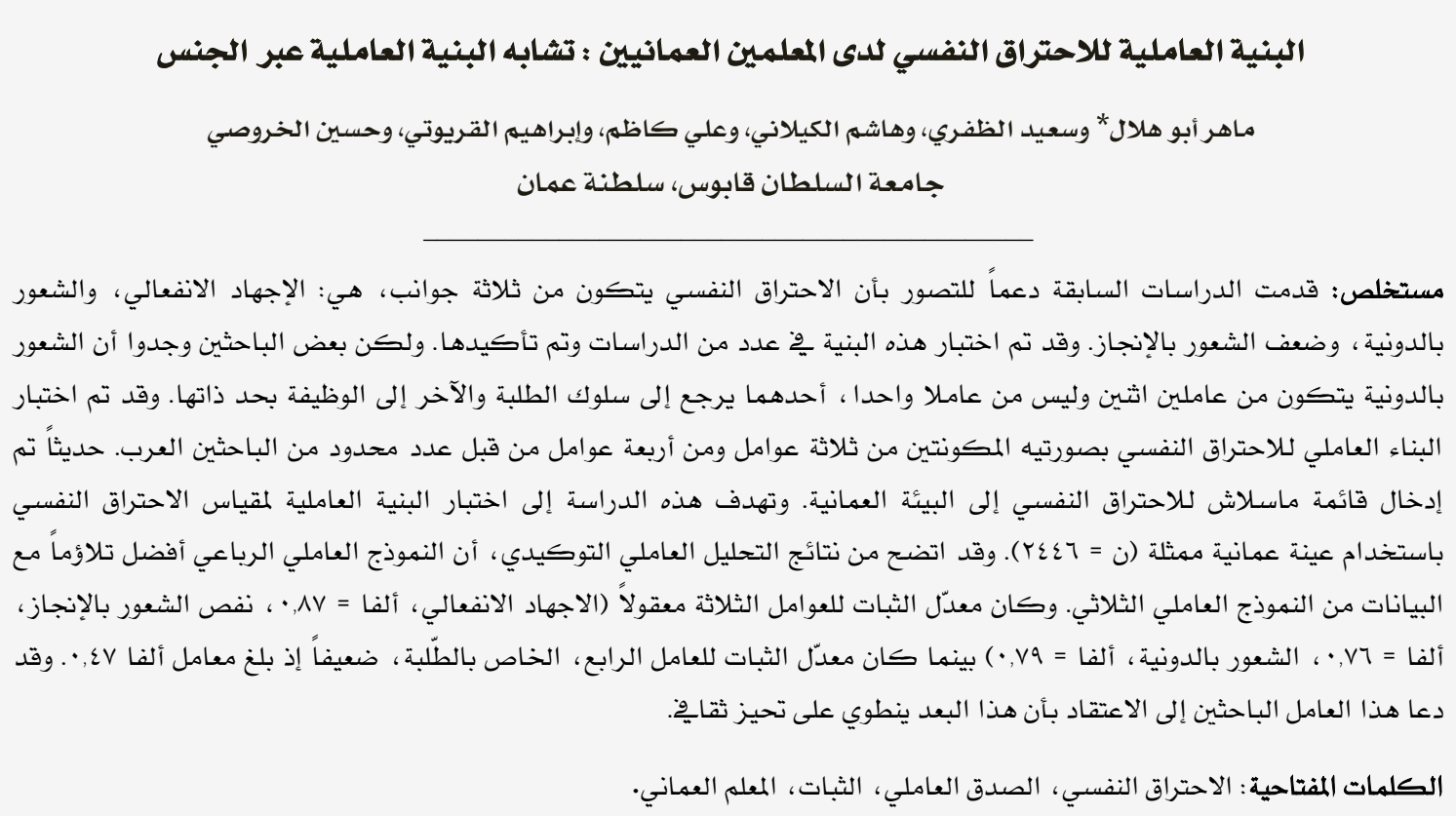

*mhilal@squ.edu.om 
As quality in teaching has been demanded by the public, more pressure has been exerted on teachers and administrators to provide the public with more efficacious outcomes. Furthermore, teachers and administrators are pressured to: accommodate the differing students, adapt the school system to these students, and contain the problems they bring with them to school. As teaching is now regarded by teachers as highly stressful (Borg, 1990), it can ultimately cause burnout among teachers. According to Farber (1991), between $5 \%$ and $20 \%$ of American teachers are burned out. As for Arab teachers, no accurate and conclusive statistics have been offered. However, practices such as absenteeism and early retirement among teachers may make us infer that such practices may result from the burnout symptom.

Research on teacher burnout and stress has received attention in many countries in recent years (Denton, Chaplin, \& Wall, 2013; Worley, Vassar, Wheeler, \& Barnes, 2008). Despite the proliferation of studies on the prevalence and sources of teacher stress and burnout (Beck \& Garguilo, 1983; Borg \& Falzon, 1989; Byrne, 1994; Chan \& Hui, 1995; Dentonet al., 2013;Starnman\& Miller, 1992), research in the Arab world, and in Oman in particular, is scarce. Additionally, the nature and structure of burnout is yet to be fully established in several Arab countries; particularly, depersonalization as one of burnout dimensions.

Several studies have investigated the structure of burnout (e.g., Abu-Hilal, 1995; Abu-Hilal \& Salameh, 1992; Byrne, 1994; Denton, et al., 2013; Iwaniki \& Schwab, 1981; Maslach \& Jackson, 1981; Richardsen, \& Martinussen, 2005; Schaufeli, Bakker, Hoognuin, Schaap, \& Kladler, 2001; Starnman \& Miller, 1992). In these studies, burnout has been conceptualized as a multidimensional construct comprising three distinct but related facets: Emotional exhaustion, depersonalization, and reduced personal accomplishment.

Emotional exhaustion has been viewed as the primary manifestation of the burnout syndrome. It has been defined as "feelings of being overextended and depleted of one's emotional and physical resources" (Halbesleben \& Demerouti, 2005, p. 208). Also, it is the most widely reported and thoroughly analyzed dimension, and considered by some researchers as the only critical component to the burnout syndrome (Skaalvik \& Skaalvik, 2010; Worley et al., 2008). However, emotional exhaustion cannot fully account for the relationship that employees have with their work. Hence, depersonalization has been recognized to take "into account the worker's detached response to either the service recipients or aspects of the job itself" (Worley et al., 2008, p. 800). Depersonalization has been characterized as a defense mechanism by which the worker places a distance from service recipients and treats them as impersonal objects to make job demands more manageable (Worley et al., 2008). Although theoretically conceptualized, empirically, it has not been fully defined.

The final dimension of burnout, personal accomplishment, refers to positive outcomes such as satisfaction and productivity in the job. It is not necessarily considered as the opposite of emotional exhaustion or depersonalization but it may correlate negatively with each of them. In several studies where personal accomplishment was measured with positively worded items, the correlations of personal accomplishment with emotional exhaustion and depersonalization were negative (see Abu-Hilal \& Salameh, 1992; Abu-Hilal, 2000).

Although many researchers have established the three-factor model of burnout, some researchers, however, have found that the threefactor model of burnout didn't fully apply to teachers (e.g., Iwaniki \& Schwab, 1981). Iwaniki and Schwab (1981) used the Maslach Burnout Inventory (MBI) to test the structure of burnout among teachers. They found four underlying dimensions rather than three. Emotional exhaustion and personal accomplishment corresponded to the first two factors identified by Maslach and Jackson (1981). However, Iwaniki and Schwab found that depersonalization broke down into two factors: (a) depersonalization of the job and (b) depersonalization of students. More recently, Denton et al. (2013) used a combination of items that measured depersonalization of the job and the students and labeled the factor 'depersonalization at the work place'. They found 4 of the 5 items valid measures of depersonalization.

On a Jordanian sample of teachers, Abu-Hilal and Salameh (1992) could replicate only depersonalization of the job. The items that measured depersonalization of students (3 items) had very small weights and did not 
make a factor of their own. Contrary to AbuHilal and Salameh (1992), Starnaman and Miller (1992) used only the items related to students to compose the depersonalization factor. They were unable to replicate the same factor with 5 items as proposed by Maslach and Jackson (1981).

In a cross-national investigation, Abu-Hilal (1995) tested the structure of burnout via confirmatory factor analysis with samples of teachers from Jordan and the United Arab Emirates (UAE). Abu-Hilal found that the structure of burnout across the two samples was invariant and the weights of items representing depersonalization of students were rather small ranging from .19 to .35 .

Both exploratory factor analysis and confirmatory factor analysis have been used to assess the dimensions of burnout and its construct validity. Worley et al. (2008) reviewed 45 studies, and reported that eleven of the studies that used exploratory factor analysis found support for an uncorrelated three-factor model. However, studies that used confirmatory factor analysis consistently showed an improved model fit with a correlated three factor solution (Worley et al., 2008). Previous literature has shown item level modifications: item deletion, and cross-loading of some items (AbuHilal \& Salameh, 1992; Starnaman and Miller, 1992); those were mostly related to the 5 items belonging to depersonalization (job and students).

Reliability analyses of the MBI consistently yield similar alpha coefficients for the emotional exhaustion: .83 to .91 , and personal accomplishment: .69 to .82 , across studies (Aluja, Blanch, and García, 2005; Worley et al., 2008). However, contrary to emotional exhaustion and personal accomplishment, reliability of depersonalization produced inconsistent results across several studies (e.g., Abu-Hilal, 1995, Abu-Hilal \& Salameh, 1992, Beck \& Garguilo, 1983, Dawani, Kaylani \& Aleyan, 1989, Iwaniki \& Schwab, 1981, Pierce \& Molloy, 1990, Starnman \& Miller, 1992,). As indexed by Cronbach's alpha, internal consistency of items measuring depersonalization has not been high. Alpha coefficients have ranged between .50 and .79 across studies. Different studies used different number of items to compute coefficient alpha. Some studies used all five items (e.g. Beck \& Garguilo, 1983,
Dawani et al., 1989, Pierce \& Molloy, 1990), some used three items (e.g. Starnman \& Miller, 1992), while some others used 2 items (AbuHilal, 1995, Abu-Hilal \& Salameh, 1992).

There is a proliferation of studies on burnout structure with western samples (Alujaet al., 2005; Green \&Walkey, 1988; Iwaniki and Schwab, 1981; Maslach\& Jackson, 1981; Worley et al., 2008), however, very few investigations have tested the structure of burnout with Arab (non-western) samples. Abu-Hilal and Salameh (1992), and Wheeler Vassar, Worley, and Barnes (2011) propose that there is a need for additional studies on measurement invariance and item level analyses, to explain divergent results across studies, professions, and cultures.

\section{The present investigation}

The present study took place in Oman, which is one of the Arabian Gulf states. Three main features characterize Oman. First, it is a traditional society with great majority as Muslems. Second, it is a heterogeneous society with different sects and ethnics living together in harmony. The people of Oman are free to practice their religion with no restrictions. Third, unlike other Gulf States (e.g., Saudi Arabia, Kuwait, Qatar and UAE), the people of Oman may work in service jobs like cleaning, driving and other handicraft jobs (e.g., mechanics, carpenters, farmers, etc.). As for gender parity, Omani women represent a good proportion of the work force. Women have occupied high rank positions such as ministers, parliament members, doctors and engineers as well as teachers. In fact, the number of women as teachers is much higher than number of men.

As an Arab-Islamic culture, Omanis are considered to be a collectivistic and patriarchal culture. In education, Omani public schools are segregated, where boys are taught by male teachers and girls are taught by female teachers. Females in collectivistic cultures are stereotyped to be more concerned with nurturance, warmth and connectedness. Males, on the other hand, are viewed to be assertive and competitive. Arab females, according to Abu-Hilal, Aldhafri, Al-Bahrani and Kamali (2015) showed more benevolence than males did. Abu-Hilal et al. (2015) reported that Arab females were more intuitive/imaginative than Arab males. Arab males expressed the think- 
ing preference more than Arab females; and females had the feeling preference more than males. As teaching is an interactive profession that deals with human beings - children - it is expected that these characteristics may have an impact and ramifications on how each gender deals with and reacts to children.

The aim of the present investigation was to test the structure of burnout on Omani teachers within this specific context. Specifically, this study addressed the following questions: First, which model represents the better fit structure, the three-factor structure (e.g., Maslach \& Jackson, 1981) or the four-factor structure (Iwaniki \& Schwab, 1981)? Second, can the structure of burnout with a higherorder factor produce good fit indices (Worley et al., 2008)? Third, is burnout structure invariant across gender? Fourth, are there significant differences between male and female teachers on the dimensions of the MBI? In other words, who is more burned out, the male teacher or the female teacher?

\section{Method}

\section{Participants}

A representative stratified sample $(\mathrm{N}=2446$; male $=997$, female $=1449$ ) of Omani teachers (targeted population around 18,000 teachers) was randomly drawn from the eleven school districts in the Sultanate of Oman. The average experience at present school was $6.16(\mathrm{SD}=$ 4.28 ), and the average workload was 15.66 (SD $=4.28$ ) classes per week.

\section{Measures}

Maslach burnout inventory (MBI) was among several other measures administered to the present sample. MBI consists of 22 items that measure three subscales: Emotional exhaustion (8 items), personal accomplishment (8 items) and depersonalization (5 items). MBI is a self-report validated measure (Abu-Hilal, 1995; Abu-Hilal \& Salameh, 1992; Maslach \& Jackson, 1981); yet, its validity and reliability with Omani samples are to be established. The items are measured on a 7-point scale ranging from 1 (never happens) to 7 (happens every day). Therefore, the higher the score, the more burned out the teacher is for emotional exhaustion and depersonalization. However, the lower the score of personal accomplishment, the more the teacher feels lacking personal accomplishment, and thus burned out.

\section{Analysis}

The data was split randomly into two halves. The first half $(\mathrm{n}=1064$, male $=393$, female $=$ 671) was subjected to exploratory factor analysis (EFA) using a principal-factors approach with Oblimin rotation. The objective of this analysis was to explore the factor pattern of Omani teachers' responses to MBI items. In order to confirm and cross validate the pattern of responses by teachers, the other half of the data $(n=1027$, male $=388$, female $=639)$ was subjected to confirmatory factor analysis (CFA). Three CFA analyses were conducted. In the first analysis three factors were specified; in the second analysis four factors were specified; and in the third, one higher-order factor was specified to underlie the factors of the best fitting model of first and second. The items designed to measure each factor were set free to load on only their appropriate factor except for one item (reference variable) which was fixed at 1.0. The variances of items and factors were uncorrelated. Factor structure was also tested across gender to test the hypothesis that burnout structure is invariant across gender.

Several fit indices were used to evaluate model fit. Among these indices are: the chi-square statistic, the comparative fit index (CFI), and the root mean square error of approximation (RMSEA) that have been recognized to be least affected by sample size (Dimitrov, 2010). An acceptable model fit is indicated by CFI values $>.90$, and when the RMSEA value is < .06 (Hu and Bentler, 1999). The most commonly used goodness-of-fit index for invariance has been the difference in chi square $\left(\Delta \chi^{2}\right)$. However, Cheung and Rensvold (2002) and Dimitrov (2010) found that chi-square is highly sensitive to large sample size. They proposed that difference in $\triangle \mathrm{CFI}$ or TLI is robust statistic for testing between-groups invariance models when the sample size is large. They suggested that a $\Delta$ CFI or TLI $<.01$ shows that the null hypothesis of invariance should not be rejected. Therefore, we used $\triangle \mathrm{CFI}$ as the statistic for the invariance tests. This type of analysis is an added value of this study. This study is among the very few studies that employed CFA technique to establish validity for the MBI, and especially with a large sample of teachers in newly researched context and population.

Reliability analyses were performed for each set of items representing the factors of MBI. 
Cronbach's alphas as estimates of internal consistency of responding (reliability) were computed for each subscale.

\section{Results}

\section{Exploratory Factor Analysis}

This analysis addresses the first question: is the four-factor model better than the threefactor model to represent the items of the MBI? With three factors extracted (not presented), $45 \%$ of the variance was explained. Also, items 5, 7 and 22 did not load on any of the factors. Comparatively, when four factors were extracted, about $50 \%$ of the variance was explained. However, items 7 and 22 still loaded on none of the factors. Both of the two items measure depersonalization. Table 1 shows the loadings of the items on their respective factors and the inter-factor correlations. Items 10 and 11 loaded on one factor (depersonalization due to job), whilst items 5 and 14 loaded on another factor (depersonalization due to students). The items - except item 20 - that measured emotional exhaustion loaded significantly on their factor and the items that measured personal accomplishment loaded significantly on their factor. The four factors were moderately correlated ranging from -.19 to .52 .

\section{Confirmatory Factor Analysis}

The responses of the second half of the sample were subjected to CFA. To address the first question, the analysis was performed three times. In the first time, three factors were specified to underlie the 22 items (model 1 in Table 2). In the second time, four factors were specified (model 2); while in the third (model 3), one-higher order factor was specified. Results of model-fitting procedures of the three models for the second random sample of Omani teachers are summarized in Table 2.

The results of the three-factor model (model 1) produced a $\chi^{2}{ }_{(183)}=954.341(\mathrm{CFI}=.887$, RMSEA $=.059)$. Eight-item solution for the emotional exhaustion and eight-item solution for the personal accomplishment scales were confirmed. The loadings of items on their appropriate factors were substantial and significant. Difficulties arose in obtaining a unidimensional scale for depersonalization. Although significant, the loadings of items 5
(.30) and 22 (.32) on depersonalization were rather small. Items 10, 11 and 14 had loadings of .83, .74, and .49 , respectively, on depersonalization. The small weights of items 5 and 22 suggest that these items may load more strongly on another factor. Therefore, the CFA was conducted with four factors. Figure 1 shows these loadings.

Table 1

Pattern of Factor Loadings with Direct Oblimin Rotation for Half of the Omani Sample of Teachers $(N=1064)$.

\begin{tabular}{|c|c|c|c|c|}
\hline \multirow{2}{*}{ Items } & \multicolumn{4}{|c|}{ Factor } \\
\hline & 1 & 2 & 3 & 4 \\
\hline Emotional Exhaustion 6 & .815 & \pm & & \\
\hline Emotional Exhaustion 8 & .775 & & & \\
\hline Emotional Exhaustion19 & .637 & & & \\
\hline Emotional Exhaustion 1 & .529 & & & \\
\hline Emotional Exhaustion 16 & .491 & & & \\
\hline Emotional Exhaustion 13 & .463 & & & \\
\hline Emotional Exhaustion 3 & .320 & & & \\
\hline Emotional Exhaustion 20 & & & .483 & \\
\hline PersonalAccomplishment17 & & .634 & & \\
\hline PersonalAccomplishment18 & & .545 & & -.318 \\
\hline PersonalAccomplishment15 & & .539 & & \\
\hline PersonalAccomplishment12 & & .526 & & \\
\hline Personal Accomplishment 21 & & .492 & & \\
\hline Personal Accomplishment4 & & .490 & & \\
\hline Personal Accomplishment9 & & .476 & & \\
\hline Personal Accomplishment 2 & & .412 & & \\
\hline Depersonalization 10 & & & .744 & \\
\hline Depersonalization 11 & & & .853 & \\
\hline Depersonalization14 & & & & .511 \\
\hline Depersonalization 5 & & & & .383 \\
\hline \multicolumn{5}{|l|}{ Depersonalization7 } \\
\hline \multicolumn{5}{|l|}{ Depersonalization 22} \\
\hline Factor & 1 & 2 & 3 & 4 \\
\hline 1. Emotional Exhaustion & 1.00 & & & \\
\hline 2. Personal Accomplishment & .192 & 1.00 & & \\
\hline 3. Depersonalization-Job & .520 & -.233 & 1.00 & \\
\hline $\begin{array}{l}\text { 4. Depersonalization- } \\
\text { students }\end{array}$ & .431 & -.230 & .480 & 1.00 \\
\hline
\end{tabular}

${ }^{ \pm}$Loadings less than .30 are omitted. 
Table 2

Test Statistics for Burnout Models for Omani Teachers

\begin{tabular}{lccccr}
\hline \multicolumn{1}{c}{ Model } & $\chi 2$ & DF & P & CFI & RMSEA \\
\hline 1) 3-factor model & 954.341 & 183 & .000 & .887 & .059 \\
2) 4-factor model & 744.025 & 180 & .000 & .917 & .051 \\
3) Hi-order 4-factor model & 744.591 & 181 & .000 & .917 & .051 \\
Independence model & 7037.032 & 231 & .000 & & \\
\hline
\end{tabular}

Note. DF: degrees of freedom; CFI: comparative fit index, RMSEA: Root mean square error of approximation.

As can be seen in Table 2 (model 2), the estimation of the model with four factors yielded a significantly improved and fitting model with a $\chi 2{ }_{(180)}=744.025(\mathrm{CFI}=.917$, RMSEA $=$ .051). The difference in $\chi^{2}$ between model 1 and model 2 was large $\left(\Delta \chi^{2}(3)=210.316\right)$ indicating that model 2 fit the data much better than model 1. Figure 1 shows the loadings and factor correlations from the CFA for the four-factor model. As shown in Figure 1, items 10 and 11 had loadings of .73 and .92, respectively, whereas items 5, 14 and 22 had substantial loadings on the other factor (.40, $.76, .32$, respectively). These loadings are consistent with the idea that depersonalization of students and depersonalization of the job are two distinct but related factors (see Abu-Hilal \& Al-Emadi, 2000; Iwaniki \& Schwab, 1981; Starnaman \& Miller, 1992). However, the validity of items 5, 14 and 22 in measuring the construct (depersonalization of students) is still somewhat weak.

An attempt was made to explore the higher order factor analysis to address the second question. The result of this analysis is shown in Table 2 -model 3. The fit indices of this model $\left(\chi^{2}(181)=744.591, \mathrm{CFI}=.917\right.$, RMSEA $=$ .051 , see Table 2) were not different from those of model 2 with four first-order factors. Hence, test of the hypothesis of invariance of structure across gender was made using the 4-firstorder factors (model 2).

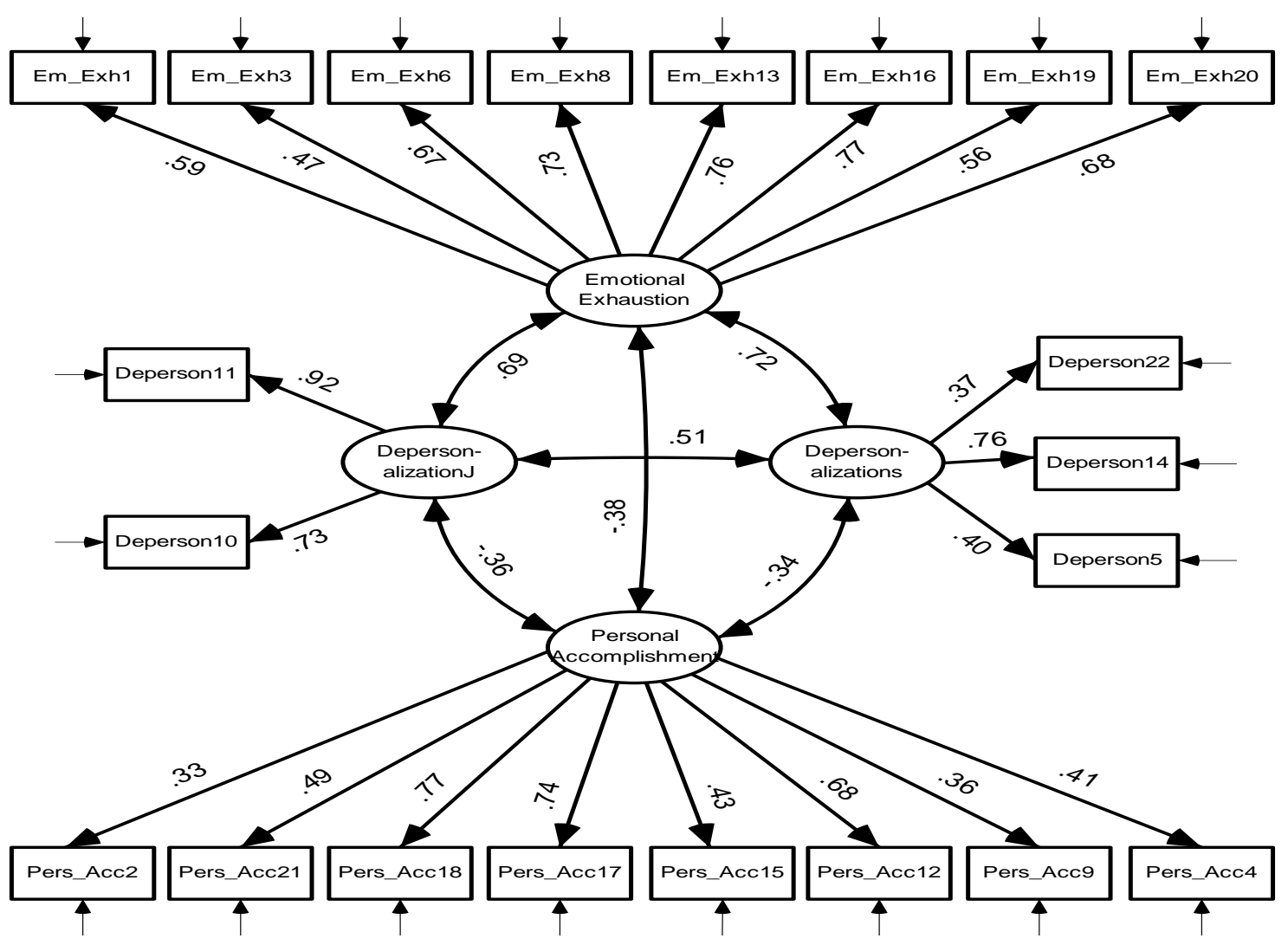

Figure 1

Loadings and Factor Correlations for a Four-Factor Model of Burnout among Omani Teachers.

Note. Numbers in rectangles represent numbers in the instrument; Depersonalization J: depersonalization of job; Depersonalizations: depersonalization of students.

All coefficients are significant, $p<.01$. 
To address the third question: 'do male and female teachers exhibit similar structure of burnout?', the second half of the dataset was subjected to CFA with invariance across gender. Table 3 shows that the fit statistics for the unconstrained model fits reasonably well the data $\left(\chi^{2}(360)=919.394\right.$, CFI $=.918$, RMSEA $=$ .036). Also, with measurement weights constrained to be equal across gender, the fit was reasonable $\left(\chi^{2}(377)=1000.779, \mathrm{CFI}=.909\right.$, RMSEA $=.037)$. However, invariance of other parameters was not adequately supported based on $\triangle$ CFI $=.032(>.01)$ between the model that constrained measurement weights and the next model in the hierarchy, measurement intercepts.

Inter-factor correlations, shown in Table 4, indicate that burnout factors were more strongly correlated for male teachers than female teachers. Specifically, emotional exhaustion and depersonalization of students were strongly related for males but less for females. This result suggests that male teachers may perceive their emotional exhaustion strongly connected with difficulties they experience when dealing with male students.

Table 3

Fit Indices of Structural Invariance across Gender

\begin{tabular}{|c|c|c|c|c|c|}
\hline Model & $\chi^{2}$ & DF & $\begin{array}{c}\text { CF } \\
\text { I }\end{array}$ & $\begin{array}{c}\text { RMSE } \\
\text { A }\end{array}$ & $\Delta \mathrm{CFI}$ \\
\hline Unconstrained & $919.394^{* *}$ & 360 & .918 & .036 & ----- \\
\hline $\begin{array}{l}\text { Measurement } \\
\text { weights }\end{array}$ & $1000.779 * *$ & 377 & .909 & .037 & .007 \\
\hline $\begin{array}{l}\text { Measurement } \\
\text { intercepts }\end{array}$ & $1206.401^{* *}$ & 398 & .882 & .041 & .032 \\
\hline $\begin{array}{l}\text { Structural } \\
\text { covariances }\end{array}$ & $1219.454^{* *}$ & 408 & .881 & .041 & .029 \\
\hline $\begin{array}{l}\text { Measurement } \\
\text { residuals }\end{array}$ & $1345.062^{* *}$ & 432 & .866 & .042 & .038 \\
\hline $\begin{array}{l}\text { Independence } \\
\text { model }\end{array}$ & $7299.626^{* *}$ & 462 & .000 & .111 & --- \\
\hline
\end{tabular}

\section{Reliability Analyses}

Internal consistency of item responses of the full sample on each of the MBI scales was the basis for estimating reliability. Coefficient alpha estimates of reliability for Omani teachers are presented in Table 5 for both male and female teachers. As can be seen in Table 5, alpha coefficients that resulted from male data for emotional exhaustion, and personal accomplishment were .88 and .79 , respectively. As for females' responses, alphas for emotional exhaustion and personal accomplishment were .86 and .72 , respectively.
These coefficients reflect measures with adequate reliability. Furthermore, correlations among items designed to measure each subscale and corrected item-scale correlations not reported here - demonstrate that every item is significantly and substantially correlated with the other items designed to measure the same facet of burnout.

Table 4

Correlations among Burnout Latent Factors for Omani Male and Female Teachers

\begin{tabular}{llccr}
\hline Factor & 1 & 2 & 3 & \multicolumn{1}{c}{4} \\
\hline 1. $\begin{array}{l}\text { Emotional ex- } \\
\text { haustion }\end{array}$ & --- & .790 & .711 & .367 \\
2. $\begin{array}{l}\text { Depersonaliza- } \\
\text { tion- student }\end{array}$ & .677 & --- & .575 & -304 \\
3. $\begin{array}{l}\text { Depersonaliza- } \\
\text { tion-job }\end{array}$ & .678 & .461 & --- & -444 \\
4. $\begin{array}{l}\text { Personal accom- } \\
\text { plishment }\end{array}$ & -.391 & -.332 & -.312 & --- \\
\hline
\end{tabular}

Note. Coefficients above diagonal for males, below for females.

All coefficients are significant at $\mathrm{p}<.001$.

As this study partially focused on depersonalization, reliability estimates were computed for items related to depersonalization of students and for items related to depersonalization of the job separately. Coefficient alphas were .47 and .79 , respectively. Alpha coefficients of depersonalization of students across gender were .48 and .40 for males and females, respectively. In contrast, alphas of depersonalization of the job across gender were .79 and .79 for males and females, respectively. In support of previous research on non-western teachers (see Abu-Hilal, 1995; Abu-Hilal \& Salameh, 1992), items related to depersonalization of students had weak internal consistency. Table 5 presents a summary of these results for male and female Omani teachers. It is noticed in Table 5 that alphas for male teachers were slightly greater than female teachers. With the exception of depersonalization - students alphas reflect reasonable internal consistency for Omani female and male teachers.

\section{Levels of Burnout}

To assess and compare the levels of burnout experienced by Omani teachers for the overall sample (fourth question), means and standard deviations related to the four facets of burnout were examined with MANOVA. MANOVA revealed a significant gender effect on the three constructs with Wilks' Lambda $(4,2117)=$ $0.912, \mathrm{~F}=51.241, p<.01$, eta squared $=.088$ ). 
Table 5

Alpha Coefficients of Burnout Subscales for Omani Male $(n=820)$ and Female $(n=1302)$ Teachers, and for Total Sample $(n=2122)$

\begin{tabular}{lccr}
\hline Factor & Male & Female & $\begin{array}{c}\text { Total } \\
\text { sample }\end{array}$ \\
\hline $\begin{array}{l}\text { Emotional exhaustion } \\
\begin{array}{l}\text { Depersonalization- } \\
\text { student }\end{array}\end{array}$ & .88 & .86 & .87 \\
$\begin{array}{l}\text { Depersonalization-job } \\
\text { Personal accomplish- }\end{array}$ & .79 & .40 & .47 \\
ment & .79 & .79 & .79 \\
\hline
\end{tabular}

A review of the between effect results, summarized in Table 6, reveal that Omani male and female teachers had more or less similar levels of burnout. Specifically, male and female Omani teachers exhibited similar feelings of emotional exhaustion. Similarly, the difference between means of depersonalization of job for females $(\mathrm{m}=4.20)$ and males $(\mathrm{m}=4.19)$ was not significant $\left(\mathrm{F}_{(1,2120)}=.005\right.$, $p>.05)$. However, the difference between the means of depersonalization of students for females $(m=6.14)$ was significantly smaller than the mean for males $\left(\mathrm{m}=7.94, \mathrm{~F}_{(1,2120)}=\right.$ $138.118, p<.01$ ), thus less burned out because of students. Also, the difference between the means of personal accomplishment for females $(\mathrm{m}=45.24)$ was significantly greater than the mean of males $(\mathrm{m}=44.03)$ as statistic $F(1,2120)=11.12, p<.01)$, indicating that females felt more personally accomplished and less burned out.

\section{Discussion}

The current study examined the structure of burnout with Omani sample. The results from the present investigation add further support to the idea that burnout is a multidimensional construct. Consistent with the earlier research of Iwaniki and Schwab (1981), the study confirmed four relatively independent dimen- sions. Our results, however, contradict the results of previous studies on non-western samples of teachers (e.g., Abu-Hilal, 1995; Abu-Hilal \& Salameh, 1992), which confirmed three burnout factors and depersonalization of students was not confirmed in either of the latter two studies.

Even with depersonalization of students clearly identified for the Omani sample, the items measuring this factor need further consideration in research with non-western samples of teachers. Correlations among items and their internal consistency suggest that depersonalization of students ought to be conceptually redefined. Consequently, both items' contents and numbers need to be reconsidered to form a more valid and reliable construct of depersonalization due to students for non-western teachers.

The similarity of responses across Omani male and female teachers indicates that burnout may be a universal phenomenon. Also, burnout's factor structure in the present investigation is remarkably consistent with western studies (see Worley et al., 2008). Furthermore, factor loadings' similarity between the two samples especially for emotional exhaustion and personal accomplishment; indicate that MBI is valid for use in research in the non-western Omani educational setting.

In factor analysis, the correlations among factors are indicative of the way constructs are differentiated and distinguished. That is, the larger the correlations the less distinguished the factors are. The factor correlations from the exploratory factor analysis produced moderate correlations in consistency with previous research in Western and non-Western settings (e.g., John, 2007).

Table 6

Means, standard deviations and F-statistics for burnout dimensions for male and female Omani teachers

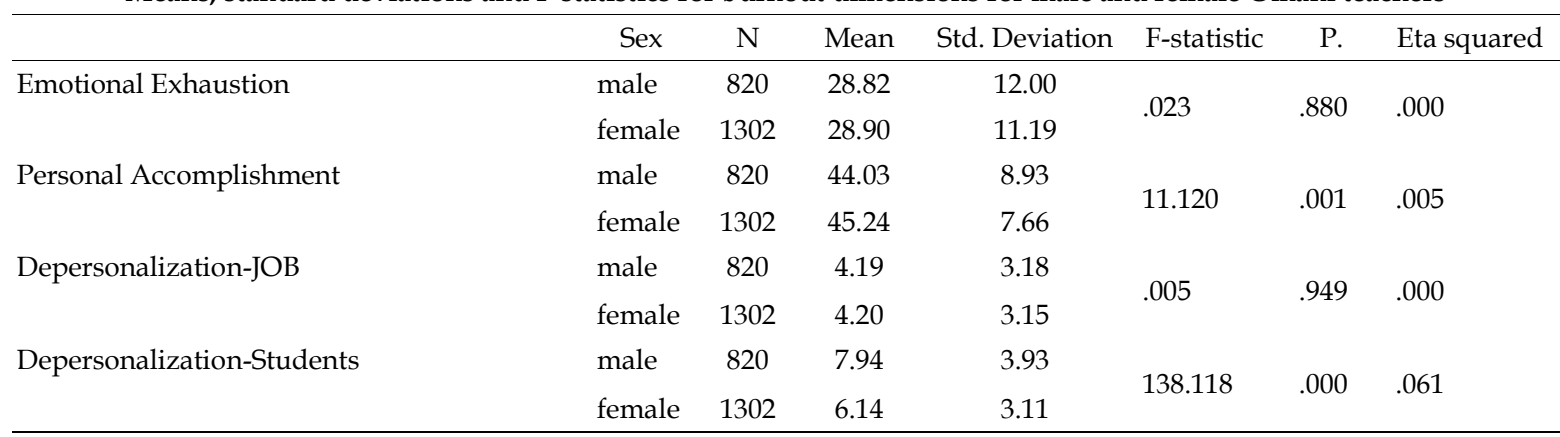


The correlations among the latent factors for male Omani teachers were larger than the correlations for female teachers, especially the two main components of burnout: emotional exhaustion and depersonalization. As inter-factor correlations are greater for male Omani teachers, they seem to be less able to distinguish between their perceptions of various aspects of burnout than female teachers do. Culturally speaking, Omani school girls have been found to be more motivated and achievement oriented than school boys (AbuHilal \& Al-Malki, 2014). These characteristics of girls make teaching tasks and duties easier and less stressful for female teachers than for male teachers. Teachers are blamed for weak achievement of boys and this may add pressure on teachers and may ultimately cause their emotional exhaustion and depersonalization.

The similarity of structure across gender provides a justification for making comparisons of means on burnout factors for the two genders. The comparisons indicate that the severity of burnout for samples of teachers in Oman is nearly the same except for personal accomplishment and depersonalization of students. These results are not surprising since Omani society is a patriarchal society. Usually men are financially responsible in the family and not women. With more demands placed on men as life style has become more complex lately, they may become more stressful and exhibit more depersonalized feelings. This is especially true if male teachers compare their economic status and income with other professions like doctors and engineers. What is economically expected from women is not much in most Arab societies (see Abu-Hilal, 2005). Any income obtained by the woman is considered a plus - but not required - for the family. Hence, female teachers showed more personal accomplishment than male teachers.

Emotionally speaking, women - as mothers are more passionate with children than men. Females in collectivistic cultures like Oman are more concerned with quality of life, nurturance, warmth and social connectedness, while males are viewed as more assertive and competitive. Given these characteristics of males and females and the nurturance nature of teaching, it may be that female Omani teachers engaged in less depersonalization than male teachers (see Abu-Hilal et al., 2015). Notwithstanding these differences across gender in Oman, the overall comparison provide support to the results of Kobasa $(1979,1982)$ that teachers -regardless of gender - may develop ways of coping with stressful situations and experience, more or less, similar levels of burnout especially with regard to emotional exhaustion which is the core of burnout.

In conclusion, the results of factor analysis, reliability analysis, and mean score comparisons support the generalizability of the Maslach burnout inventory for male and female Omani samples, notwithstanding that minor differences have occurred across the two samples. The overall picture of interfactor correlations provides support to discriminant validity of MBI. Nevertheless, researchers in Oman and in other Arab - non Western - populations are encouraged to further evaluate the depersonalization dimension of burnout. Special emphasis should be directed to depersonalization of students. Probably, more qualitative and in-depth research needs to be done. Also, experimental designs can be employed to test relationship between emotional exhaustion and depersonalization. The latter facet of burnout needs to be more thoroughly investigated and reformulated.

\section{References}

Abu-Hilal, M. (1995). Dimensionality of burnout: Testing for invariance across Jordanian and Emirati teachers. Psychological Report, 77, 1367-1375.

Abu-Hilal, M., \& Al-Malki, H. (2014).Frame of reference and achievement across gender among Omani middle school students. International Journal of Psychological and Educational Assessment, 16(2), 8199.

Abu-Hilal, M., Aldhafri, S., Al-Bahrani, M., \& Kamali, M. (2015). The Arab Culture and the Arab Self: Emphasis on Gender. In R. King\& Allan Bernardo (Eds.). The psychology of Asian learners (pp. 125-138). Singapore: Springer.

Abu-Hilal, M., \& Al-Emadi, A. (2000). Factor structure and reliability of burnout 
among Emirati and Palestinian teachers. Psychological Reports, 87, 597-610.

Abu-Hilal, M., \& Salameh, K. (1992). Validity and reliability of the Maslach burnout inventory for non-Western teachers. Educational and Psychological Measurement, 52, 161-169.

Aluja, A., Blanch, A., \& García, L.F. (2005). Dimensionality of the Maslach burnout inventory in school teachers: A study of several proposals. European Journal of Psychological Assessment, 21(1),67-76.

Beck, C., \& Garguilo, R. (1983). Burnout in teachers of retarded and nonretarded children. Journal of Educational Research, 76, 169-173.

Borg, M. G. (1990). Occupational stress in British educational settings: A review. Educational Psychology, 10, 103-126.

Borg, M. G., \& Falzon J. M. (1989). Stress and job satisfaction among primary school teachers in Malta. Educational Review, 41, 271-279.

Byrne, B. M. (1994) Burnout: Testing for validity, replication, and invariance of causal structure across elementary, intermediate and secondary teachers. American Educational Research Journal, 31, 645-673.

Chan, D. W., \& Hui, E. K. (1995) Burnout and coping among Chinese secondary school teachers in Hong Kong. British Journal of Educational Psychology, 65, 1525.

Cheung, G. W., \& Rensvold, R. B. (2002). Evaluating goodness-of-fit indexes for testing measurement invariance. Structural Equation Modeling, 9, 233-255. DOI:10.1207/S15328007SEM0902_5

Dawani, K., Kaylani, A., \& Alayan, K. (1989). Levels of burnout among public school teachers in Jordan. Educational Journal, 5, 253-273.

Denton, E., Chaplin, W. F., \& Wall, M. (2013). Teacher burnout: A comparison of two cultures using confirmatory factor and item response models. International Journal of Quantitative Research in Education, 1(2), 147-166.
Dimitrov, D. M. (2010). Testing for factorial invariance in the context of construct validation. Measurement and Evaluation in Counseling and Development, 43, 121149.DOI: $10.1177 / 0748175610373459$

Halbesleben, J. R., \& Demerouti, E. (2005). The construct validity of an alternative measure of burnout: Investigating the English translation of the Oldenburg Burnout Inventory. Work and Stress, 19, 208-220.

Hu, L. T., \& Bentler, P. M. (1999). Cutoff criteria for fit indexes in covariance structure analysis: Conventional criteria versus new alternatives. Structural Equation Modeling, 6, 1-55. doi: 10.1080/10705519909540118

Iwaniki, E. F., \& Schwab, R. L. (1981). A cross validation study of the Maslach Burnout Inventory. Educational and Psychological Measurement, 41, 1167-1174.

John, G. J. (2007). An exploratory study of the prevalence and nature of burnout among public primary and secondary qualified school teachers in Saint Lucia. (Unpublished master thesis, Trinity Western University).

Kobasa, S. C. (1979). Stressful life events, personality, and health: An inquiry into hardiness. Journal of Personality and Social Psychology, 37, 1-11.

Kobasa, S. C. (1982). Commitment and coping in stress resistance among lawyers. Journal of Personality and Social Psychology, 42, 707-717.

Maslach, C., \& Jackson, S. (1981). The measurement of experienced burnout. Journal of Occupational Behavior, 2, 99-113.

Pierce, C. M., \& Molloy, G. N. (1990). Psychological and biographical differences between secondary school teachers experiencing high and low levels of burnout. BritishJournal of Educational Psychology, 60, 37-51.

Richardsen, A. M., \& Martinussen, M. (2005). Factorial validity and consistency of the MBI-GS across occupational groups in Norway. International Journal of Stress Management, 12(3), 289-297. 
Schaufeli, W. B., Bakker, A. B., Hoognuin, K., Schaap, C., \& Kladler, A. (2001). On the clinical validity of the Maslach Burnout Inventory and the burnout measure. Psychology and Health, 16, 565-582.

Skaalvik, E. M., \& Skaalvik, S. (2010). Teacher self-efficacy and teacher burnout: A study of relations. Teaching and Teacher Education, 26, 1059-1069. doi:10.1016/j.tate.2009.11.00

Starnman, S. M., \& Miller, K. I. (1992). A test of causal model of communication and burnout in the teaching profession. Communication Education, 41, 40-53.

Wheeler, D.L., Vassar, M., Worley, J.A., \& Barnes, L.L.B. (2011) A reliability generalization meta-analysis of coefficient alpha for the Maslach burnout inventory. Educational and Psychological Measurement, 71(1), 231-244.

Worley, J. A., Vassar, M. Wheeler, D. L., \& Barnes, L. B. (2008). Factor structure of scores from the Maslach Burnout Inventory: A review and meta-analysis of 45 exploratory and confirmatory factoranalytic studies. Educational and Psychological Measurement, 68, 797-823.doi: 10.1177/0013164408315268 